Math. Model. Nat. Phenom.

Vol. 4, No. 4, 2009, pp. 118-130

DOI: $10.1051 / \mathrm{mmnp} / 20094404$

\title{
In vitro Vasculogenesis Models Revisited - Measurement of VEGF Diffusion in Matrigel
}

\author{
T. Miura ${ }^{1,2 *}$ and R. Tanaka ${ }^{3}$ \\ ${ }^{1}$ Department of Anatomy and Developmental Biology \\ Kyoto University Graduate School of Medicine \\ ${ }^{2}$ JST CREST \& PRESTO \\ ${ }^{3}$ Department of Mathematics, Kyoto University Faculy of Science.
}

\begin{abstract}
The circulatory system is one of the first to function during development. The earliest event in the system's development is vasculogenesis, whereby vascular progeniter cells form clusters called blood islands, which later fuse to form capillary networks. There exists a very good in vitro system that mimics this process. When HUVECs (Human Umbilical Vein Endothelial Cells) are cultured on Matrigel, they spontaneously form a capillary network structure. Two theoretical models have been proposed to explain the pattern formation of this in vitro system. Both models utilize chemotaxis to generate spatial instability, and one model specifies VEGF as the chemoattractant. However, there are several unknown factors concerning the experimental model. First, the pattern formation process occurs at the interface between the liquid medium and Matrigel, and it is unclear whether diffusion in the liquid or gel is critical. Second, the diffusion coefficient of VEGF, which determines the spatial scale of the capillary structure, has not been properly measured. In the present study, we modified the experimental system to clarify the effect of diffusion in Matrigel, and experimentally measured the diffusion coefficient of VEGF in this system. The relationship with the spatial scale of the pattern generated is discussed.
\end{abstract}

Key words: pattern formation, HUVEC, vascular endothelial growth factor (VEGF), diffusion, Matrigel

AMS subject classification: 92B05

${ }^{*}$ Corresponding author. Email: miura_takashi@mac.com 


\section{Introduction}

The circulatory system is one of the first to function during development [1,2]. It first appears as a collection of blood islands in the yolk sac. These blood islands then fuse to form a meshwork structure via a process called vasculogenesis. Later, new branches sprout from preexisting blood vessels to form finer vascular networks, a process known as angiogenesis. This process has been studied extensively as a target of anti-cancer therapy, since cancerous tissue attracts blood vessels to meet the demand for oxygen and nutrition of rapidly growing cells [3].
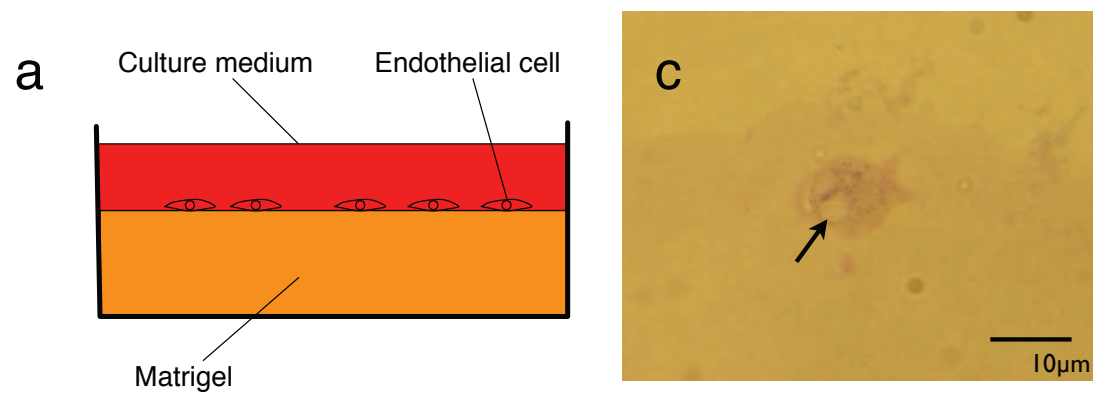

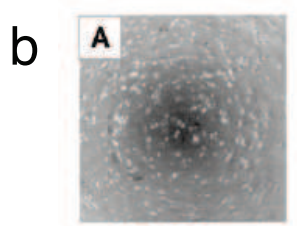

0 hour

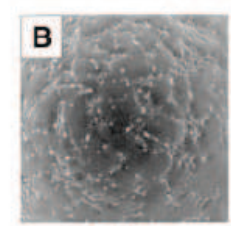

3 hours

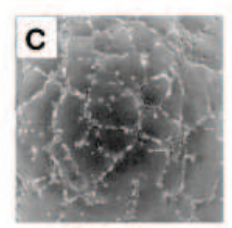

6 hours

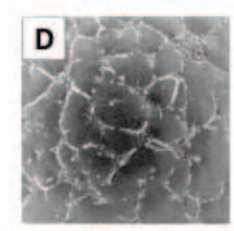

9 hours

Figure 1: In vitro vasculogenesis experiment. (a) Experimental setup. HUVECs (Human umbilical vein endotelial cells) are cultivated on Matrigel. (b) The cultured cells form a mesh-like structure within 24 hour (from [7]). (c) The structure has a lumen inside the tube (Arrow).

There is a very simple in vitro system that reproduces the vasculogenesis process [4]. When human umbilical vein endothelial cells (HUVECs) are plated sparsely on Matrigel (a protein mixture secreted by mouse tumor cells), they grow thin processes and become interconnected, which results in a meshwork structure on the gel. This system has been used to assay the effects of various chemical or hydrodynamic factors [4].

There have been several attempts to model the behavior of this experimental system. The classic model [5] assumes that the mechanical properties of Matrigel are the key to pattern formation. According to this model, cells have the ability to deform the extracellular matrix gel. As they deform the gel independently, they move passively according to the deformation, resulting in the formation of clusters of cells similar in size to the distance of effective deformation. However, the discovery of vascular endothelial growth factor (VEGF), an extracellular signaling molecule that acts as a very strong chemoattractant for endothelial cells, makes this model sound less realistic. Recent models $[6,7]$ incorporate the effect of such a chemoattractant as a crucial factor for pattern 
formation. Each endothelial cell secretes a chemoattractant and moves according to a chemoattractant gradient. As a result, areas with a slightly higher cell density have higher chemoattractant concentrations. Then the cells migrate into such areas by chemotaxis, resulting in a still higher cell density. This process results in the formation of cell clusters comparable in size to the chemoattractant gradient. Merks et al. [6] utilized the cellular Potts model to describe this phenomenon and emphasized the effect of cell shape, while Serini et al. [7] formulated a continuous model which can be handled analytically.

Based on previous studies, we now have very good working hypotheses on the mechanism of pattern formation in this in vitro system. The obvious next step is the measurement of model parameters and experimental verification of the models, which has not been done quantitatively for several reasons. First, developmental systems are highly context-dependent and measurements cannot be extrapolated to other systems, which reduces the generality of measurements. Second, a measurement method at the tissue level has not been established properly. Thanks to recent advances in Green Fluorescent Protein (GFP) technology and imaging methods, we have a number of sophisticated ways to estimate diffusion coefficients at the subcellular level [8]. However, such methods are not very useful on a larger scale, so we first need to develop a method of measurement.

In the present study, we try to measure the diffusion coefficient of VEGF in Matrigel. VEGF is one of the key factors determining the spatial scale of the meshwork structure. Our current measurement is $1.0 \times 10^{6} \mu \mathrm{m}^{2} / \mathrm{h}$, which seems too large compared to the timescale of other reactions (hour) and spatial scale of the structure $(100 \mu \mathrm{m})$. We need to incorporate other factors that can generate a structure of correct size if the measurement is to be accurate.

\section{Materials \& Methods}

\subsection{HUVEC culture}

HUVECs were purchased from CAMBREX Inc. and cultivated according to a standard protocol with EGM-2 culture medium (Cambrex). To limit the effect of diffusion via the liquid medium, we embedded the entire system in Matrigel (Fig. 2). The cells were cultured to a confluent state on glass-bottom dishes (Asahi glass inc.). Then the culture medium was removed, and $100 \mu l$ of Matrigel solution was applied to the dish. The gel was allowed to solidify by incubating at $37^{\circ} \mathrm{C}$ in a humidified atmosphere. Culture medium was added and the cells were cultured for another 24-48 hours.

\subsection{Preparation of fluorescently-labeled VEGF}

VEGF165 was purchased from Peprotech Inc. Fluorescently-labeled VEGF was prepared using an Alexa-488 microscale protein labeling kit (Molecular Probes). The labeled protein was isolated from the remaining unbound dye using a gel filtration column. The purity of the labeled protein was confirmed using SDS gel electrophoresis. 

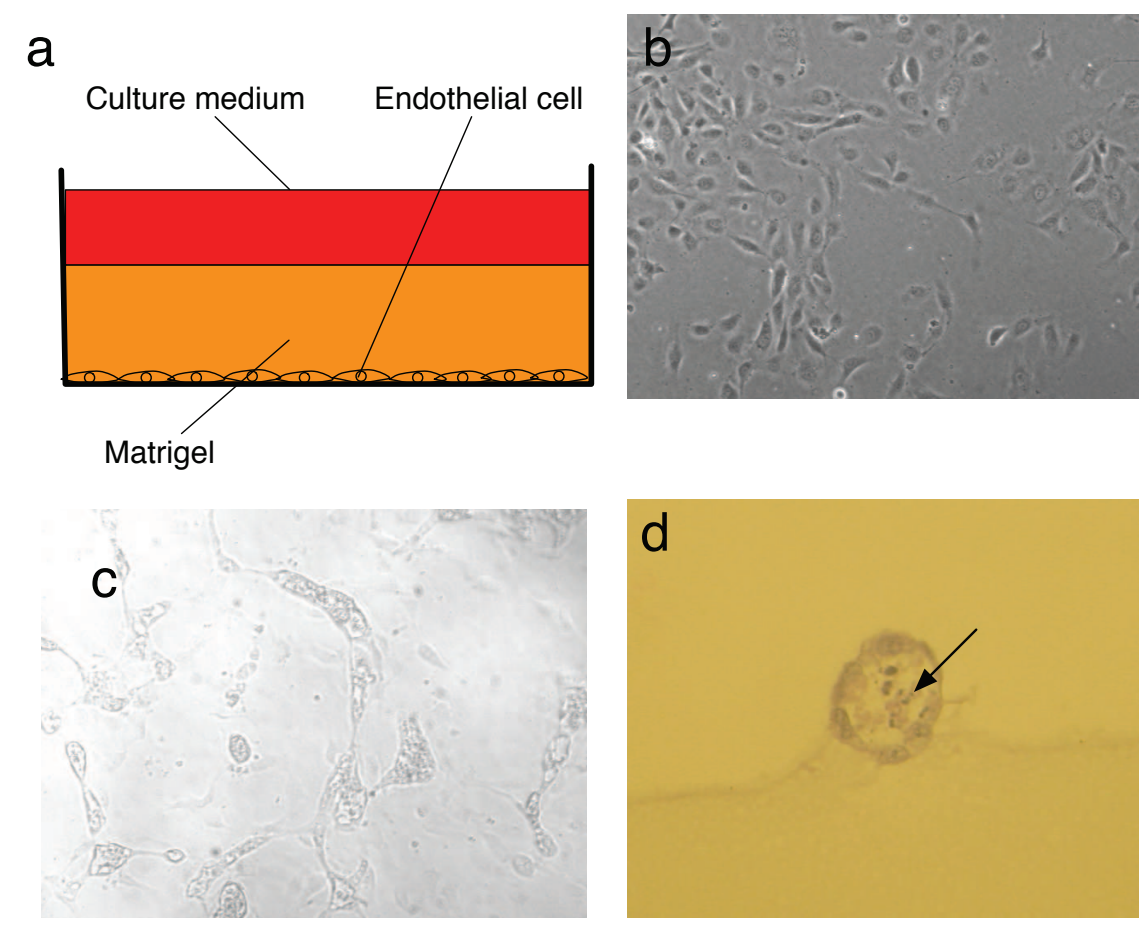

Figure 2: (a) Matrigel overlay culture method. Since the effective diffusion coefficient at the interface of the gel and liquid is difficult to determine, we embedded the HUVECs in Matrigel. Under this experimental condition, the diffusion of VEGF in Matrigel should determine the final spatial scale. (b) HUVECs before the Matrigel overlay. (c) The embedded cells formed a mesh-like structure after 7 days. The characteristic length of the meshwork structure is 100-200 $\mu \mathrm{m}$, which is not very different from previous experiments on Matrigel. (d) HE-stained section of the newly generated structure. The lumen is formed inside the tubular structure. 


\subsection{Measurement of diffusion in Matrigel by a small channel}

A thin layer $(150 \mu \mathrm{m})$ of Matrigel was prepared by assembling a slideglass and three $18 \times 18 \mathrm{~mm}$ coverglasses (Fig. 3). Then by sliding one of the slideglasses about $500 \mu \mathrm{m}$, we prepared a small channel in which we could observe "quasi-one-dimensional" diffusion dynamics. We placed the apparatus on an inverted microscope, applied a fluorescently-labeled VEGF, and captured timelapse images to obtain the diffusion dynamics of VEGF in Matrigel. The applied VEGF solution filled the channel quickly (within $1 \mathrm{sec}$ ) and variation in the VEGF concentration was minimal as seen in Fig. 3.

\subsection{Measurement of diffusion in Matrigel by fluorescence recovery after photobleaching (FRAP)}

A thin layer of Matrigel containing fluorescently-labeled VEGF was prepared. After the gel had solidified, the slide was placed on a confocal microscope (Nikon C1). Then a certain region of the gel was exposed to the highest available energy laser, and the recovery of fluorescence was observed at certain time points. The samples were kept at $37^{\circ} \mathrm{C}$ in a humidified atmosphere.

\section{Results}

\subsection{Theoretical estimate of generated spatial scale}

Serini et al. [7] suggested that the spatial scale of the generated structure should be on the order of

$$
\sqrt{D \tau}
$$

where $D$ is the diffusion coefficient of VEGF in Matrigel and $\tau$ is the characteristic time scale of VEGF decay. An intuitive explanation is as follows: in this model, the size of the generated pattern is determined by the size of the VEGF gradient in the Matrigel region. In the standard source-diffusion-degradation (SDD) model, the profile of the morphogen gradient is governed by

$$
\begin{aligned}
\frac{\partial u}{\partial t} & =D \frac{\partial^{2} u}{\partial x^{2}}-u / \tau \\
D \frac{\partial u}{\partial x} & =\phi \quad \text { at } x=0
\end{aligned}
$$

where $u$ is the VEGF concentration and $\phi$ is morphogen production rate at $x=0$. In this system, a stable gradient at $t \rightarrow \infty$ is described as

$$
u(x)=\phi \sqrt{D \tau} e^{-\frac{x}{\sqrt{D \tau}}} .
$$


In this gradient, when $x$ is multiplied by $\sqrt{D \tau}$, the concentration $u$ decreases by a factor of $1 / e$. Therefore, the size of the VEGF gradient is of the order of $\sqrt{D \tau}$. The pattern generated by the models should also be of the order of $\sqrt{D \tau}$.

We do not have definitive data on the VEGF decay speed, but can estimate the order based on common sense. When using protein molecules, we reconstitute them in an appropriate buffer and keep them at $4{ }^{\circ} \mathrm{C}$. The activity of a protein in general will not be lost within 10 min or so at room temperature. However, leaving the protein solution for 24 hours at room temperature will affect the result. Therefore, the timescale of the VEGF decay should be 1-10 hours. The characteristic spatial scale of the generated endothelial network is around 100-200 $\mu \mathrm{m}$, so the diffusion coefficient should be of the order of $1 \times 10^{4} \mu \mathrm{m}^{2} /$ hour.

\subsection{Matrigel overlay culture method}

The conventional HUVEC pattern formation assay was done on Matrigel, and it is not clear whether the diffusion coefficient in liquid medium or in gel is effective for the pattern formation process. To distinguish between these possibilities, we reproduced the pattern formation process inside the Matrigel. It has been reported that epithelial cells form a meshwork structure when embedded in collagen gel [9]. Such a structure might also be formed with HUVECs.

When we added Matrigel to the confluent HUVEC layer, the cells spontaneously formed a meshwork structure within 24 hours. Generally, tube structures were thicker than those formed on the Matrigel, and mesh size itself was comparable to that formed on the gel. Under these conditions, cells were completely embedded inside the Matrigel, so the diffusion coefficient inside the Matrigel is important.

\subsection{Measurement of VEGF diffusion using a small channel}

We then tried to estimate the diffusion coefficient in Matrigel using a small chamber (Fig. 3). When the fluorescently-labeled VEGF solution was applied to the chamber, fluorescently-labeled molecules started to diffuse inside the thin layer of Matrigel. Judging from the concentration profile (Fig. 3d), VEGF diffusion is slower in Matrigel than in solution. We could not detect an accumulation of VEGF at the interface between the gel and solution [10], indicating the effect of the extracellular matrix (ECM) trap in this case is not very important. A gradient of $1 \mathrm{~mm}$ formed within 10 minutes. This means that the diffusion coefficient of VEGF is roughly $1.0 \times$ $10^{6} \mu \mathrm{m}^{2} /$ hour, which is not very different from the general diffusion coefficient of proteins in liquid.

\subsection{Measurement of VEGF diffusion using FRAP}

We tried to observe the diffusion dynamics in a different way. One conventional method of measuring diffusion coefficients is fluorescence recovery after photobleaching (FRAP). The principle of this method is as follows: a fluorescent dye loses color when exposed to a very high energy laser beam. This phenomenon is called photobleaching. By using a confocal microscope, we can 

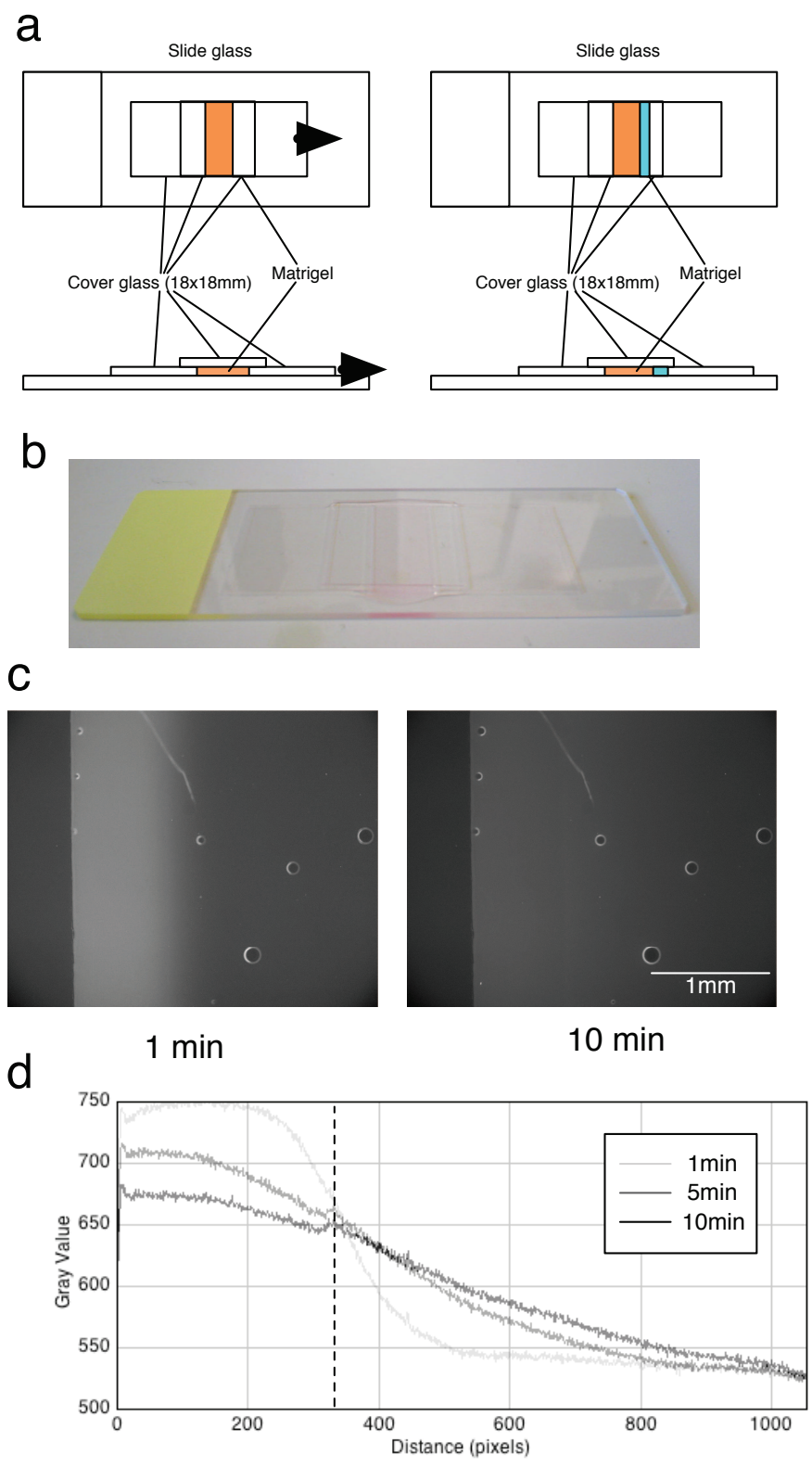

Figure 3: Direct measurement of the diffusion coefficient of VEGF in Matrigel. (a) Experimental setup. A thin layer of Matrigel was prepared using three coverslips and a slideglass, and a narrow channel was created by sliding one coverslip slightly. (b) Actual experimental apparatus. (c) Time-course observation of the diffusion process. Alexa Fluor-labeled VEGF was applied to the channel, and the diffusion of the fluorescent molecule was observed directly. A gradient of $1 \mathrm{~mm}$ is generated within 10 minutes, which shows that the diffusion coefficient of VEGF in Matrigel is in the order of $1.0 \times 10^{6} \mu \mathrm{m}^{2} /$ hour. (d) Concentration profile of the fluorescence. Dashed line indicates the boundary between solution and Matrigel. 
bleach a very narrow area in a view. When a diffusible molecule is photobleached, fluorescent molecules outside the photobleached area gradually diffuse into the area, resulting in a recovery of fluorescence.

We detected the recovery of fluorescence using FRAP (Fig. 4). The area of the bleached spot was $1000 \mu^{2}$ and the characteristic time for recovery was around $10 \mathrm{sec}$, indicating that the diffusion coefficient was of the order of $1.0 \times 10^{6} \mu \mathrm{m}^{2} /$ hour. This value is consistent with that obtained by the small channel experiment.

For a positive control experiment, we used the same fluorescent protein to determine the diffusion coefficient at the cell surface (Fig. 5). The bleached area is clearly visible, and recovery time is considerably longer than that in gel. The characteristic length of the bleached area is $10 \mu \mathrm{m}$ and recovery time is of the order of minutes, so the diffusion coefficient is roughly $1.0 \times 10^{4} \mu \mathrm{m}^{2} / \mathrm{hour}$, which is much smaller than that in Matrigel.

\section{Discussion}

In general, the diffusion coefficient of protein molecules in liquid is of the order of $1.0 \times 10^{-6} \mathrm{~cm}^{2} / \mathrm{s}$ $\left(\approx 1.0 \times 10^{6} \mu \mathrm{m}^{2} /\right.$ hour $)$. This intuitively means that a molecule moves $10 \mu \mathrm{m}$ per second. To generate a gradient of the order of $100 \mu \mathrm{m}$, the timescale of protein decay should be around 10 seconds. In this specific case the protein decay time is about 1-10 hours. Therefore, the observed diffusion coefficient is too large and we need some mechanism to slow down the diffusion. This kind of estimation has been done in a different model by [11].

There are various ways to decrease diffusion coefficients in biological tissue [12]. One possible mechanism is absorption by the extracellular matrix. One of the best studied extracellular matrices playing this role is heparan sulfate proteoglycan (HSPG), which has polysaccharide side chains which can trap extracellular signaling molecules [13].

If we assume the free and HSPG-bound VEGF concentrations to be $u(x, t)$ and $w(x, t)$ respectively, and the diffusion coefficient of VEGF to be $D$, then the overall diffusion process can be written as follows:

$$
\begin{aligned}
u^{\prime} & =\nabla \cdot D(\nabla u)-k_{a} u+k_{d} w \\
w^{\prime} & =k_{a} u-k_{d} w
\end{aligned}
$$

where $k_{a}$ and $k_{d}$ are association and dissociation constants. If we assume that the associationdissociation reaction is fast enough, we can treat $w^{\prime}$ as 0 and obtain

$$
u+w=\frac{k_{a}+k_{d}}{k_{d}} u
$$

If we add both sides of equation 4.1, we obtain

$$
(u+w)^{\prime}=\nabla \cdot D(\nabla u)=\Delta\left(D \frac{k_{d}}{k_{a}+k_{d}}(u+w)\right)
$$


a

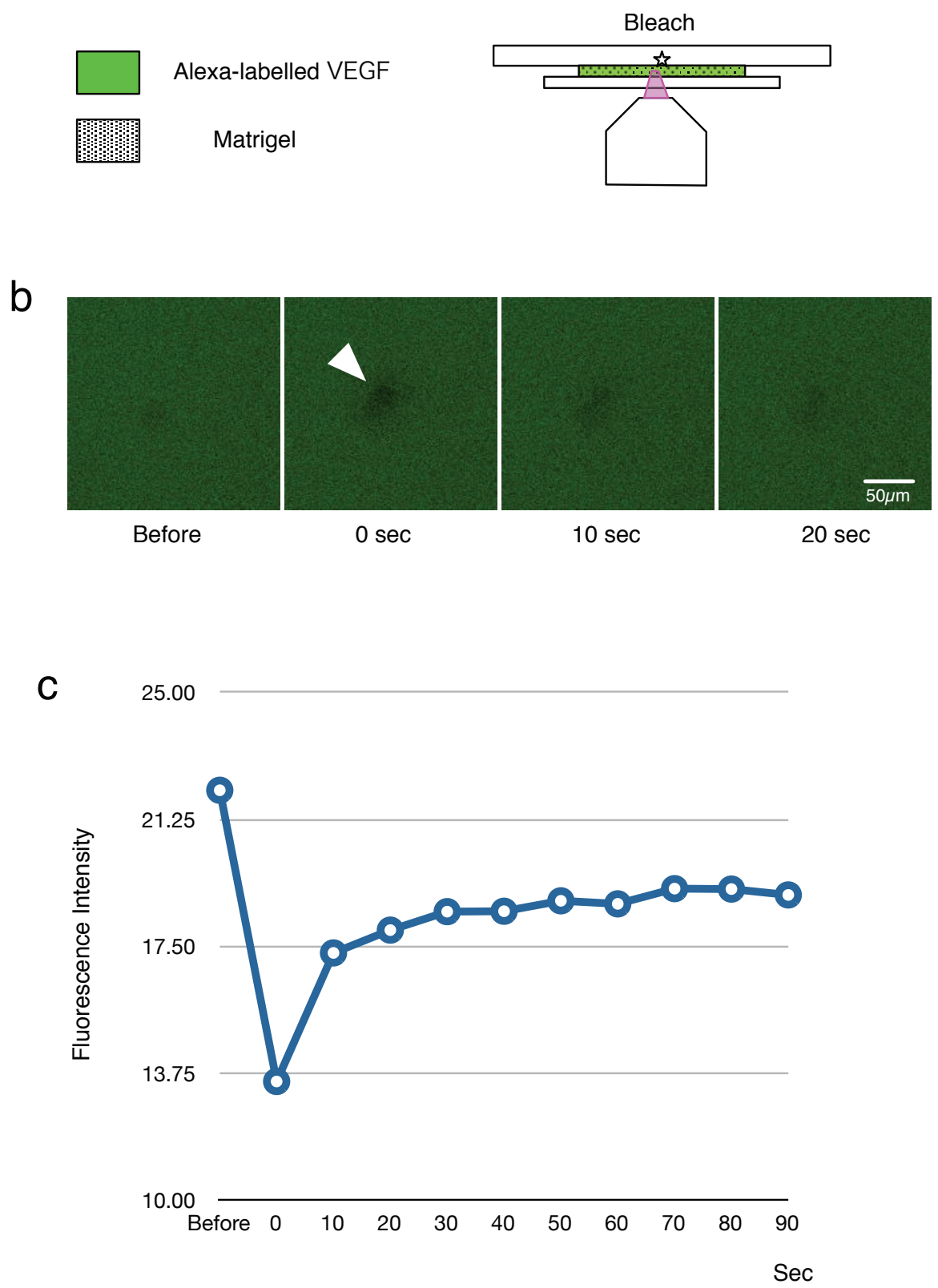

Figure 4: Measurement of the diffusion of VEGF in Matrigel using FRAP. (a) Experimental setup. A thin layer $(6 \mu \mathrm{m})$ of Matrigel containing Alexa-labeled VEGF was prepared, and photobleached by confocal microscope. (b) Time course of bleached spot. The bleached spot is indicated by a white arrowhead. (c) Change of fluorescence during the experiment. The fluorescence did not recover completely, probably due to an immobile component (for example, autofluorescence of Matrigel). 
a
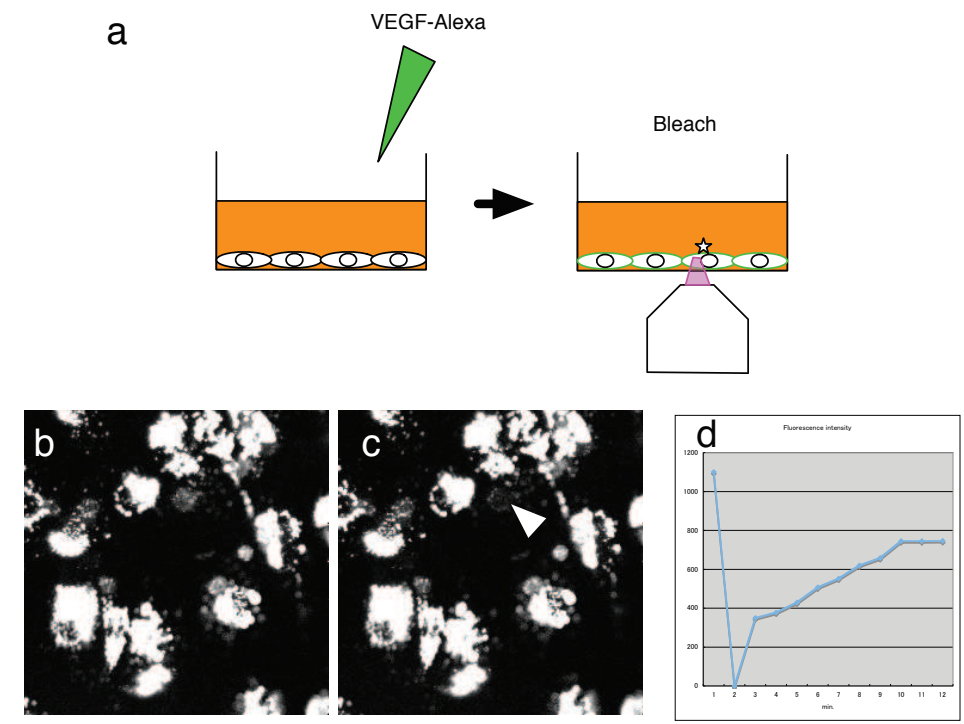

Figure 5: Measurement of VEGF diffusion on HUVECs using FRAP. (a) Experimental setup. Alexa Fluor-labeled VEGF was added to the culture medium. After 30 minutes the VEGF is localized at the cell surface. The fluorescent VEGF on the cell surface was bleached. (b) Before bleaching. (c) After bleaching. We could observe the bleached area. (d) Fluorescence recovery curve.

If we denote $u+w$ as $U$ and the effective diffusion coefficient of $U$ as $D_{e}$, the equation becomes

$$
U^{\prime}=\Delta\left(D_{e} U\right)
$$

where

$$
D_{e}=D \frac{k_{d}}{k_{a}+k_{d}} .
$$

This means when the association of a diffusible chemical with the extracellular matrix is strong, the effective diffusion coefficient decreases. This form is slightly different from the conventional Fickian diffusion coefficient, which is studied in mathematical ecology under a different context $[14,15]$. For example, if we apply the molecule in liquid to the extracellular matrix region, which is similar to the situation in Fig. 3, a very high concentration region should appear at the interface between the liquid and extracellular matrix. We experimentally observed this phenomenon in FGF diffusion [10] but not in this system, indicating that HSPG trapping of VEGF is not as strong as that of FGF.

The other mechanism is tissue structure. When cells are tightly packed, the molecules need to travel longer to move a certain distance, resulting in a decrease in the effective diffusion coefficient. The effect of tissue structure on epidermal growth factor (EGF) has been studied in detail $[16,17]$. The effect depends on tissue geometry and is very difficult to define. In this case, we can obtain electron microscopic data on the Matrigel mesh [18], but it is still not clear whether the structure of the fixed material actually represents the real structure. 
Another possible mechanism is the uptake of growth factors via cell surface receptors. The uptake of VEGF by HUVECs can be assayed using fluorescently-labeled VEGF and we have preliminary data indicating that the process is relatively fast (around 10 minutes), which may help to generate the small structure in vivo. However, in this in vitro system, there are no cells between clusters and uptake should not have an effect. The effect of various factors on the diffusion of extracellular signaling molecules was reviewed in [12].

The sensitivity of cells to a morphogen gradient might affect the spatial scale of generated structure. In Dictyostelium chemotaxis studies, the mechanism of sensing a shallow gradient has been studied in detail [19]. The sensitivity of HUVECs to an external VEGF gradient has been measured quantitatively using a microdevice [20], but the situation might be different in Matrigel.

Although this culture system generates patterns similar to those observed in vivo, we need to be cautious when extrapolating this result to the situation in vivo. This in vitro system only contains cells that form blood vessels, and does not reproduce signals from hypoxic tissue. Therefore, the VEGF concentration in vivo may be higher in surrounding tissues, while in this system, the concentration is high around the tube structure. The situation In vivo has been modeled extensively in neovascularization to tumor tissue [3].

Merks et al. [6] pointed out that VEGF165 is not a proper candidate for a morphogen in this system because the diffusion coefficient is too large, which is consistent with our observation. They argued that VEGF185, a larger splicing variant, may play a role. There is experimental evidence that a larger splicing variant of VEGF is trapped by ECM [21], and a smaller VEGF splicing variant generates a larger capillary meshwork in vivo [22].

We still can not rule out the possibility that this large diffusion coefficient comes from measurement error. For example, we used a relatively high concentration of VEGF for visualization purposes, which might have affected the experimental result. If the binding site of VEGF in the ECM is saturated, the effect of buffering in the ECM should be less evident than the actual biological situation. Another possibility is the labeling method itself. We used the Alexa fluor 488 label for visualization. The Alexa dye has stronger fluorescence and a smaller molecular weight than green fluorescent protein (GFP), but might be covalently linked to multiple residues and block binding to the extracellular matrix.

The present study shows that even a very rough quantitation of parameters can be useful in validating theoretical models. Traditionally, mathematical modeling of biological systems has started with the nondimensionalization of parameters [3], and mainly tried to derive qualitative predictions which do not depend on actual parameters. This tradition is due to a lack of quantitative data in molecular biology, but with advances in imaging techniques, we are beginning to obtain the quantitative data necessary for evaluating models. In addition, experimental biologists actually have a sense of scale for the system in many cases, and utilizing such information can be very useful. 


\section{Acknowledgements}

The authors are grateful to Yoshimi Yamaguchi for technical assistance. This work was financially supported by JST CREST, PRESTO and JSPS.

\section{References}

[1] S. Gilbert. Developmental Biology. Sinauer, Massachusettes, 2003.

[2] T. Sadler. Langman's Medical Embryology. Lippincott Williams \& Wilkins, Maryland, 9th edition, 2004.

[3] J. Murray. Mathematical biology. Springer - Verlag, Berlin, third edition, 2003.

[4] W. Aird. Endothelial Biomedicine. Cambridge university press, 2007.

[5] D. Manoussaki, S. Lubkin, R. Vernon, J. Murray. A mechanical model for the formation of vascular networks in vitro. Acta Biotheor, 44 (1996) No. 3-4, 271-282.

[6] R. Merks, S. Brodsky, M. Goligorksy, S. Newman, J. Glazier. Cell elongation is key to in silico replication of in vitro vasculogenesis and subsequent remodeling. Dev Biol, 289 (2006) No. 1, 44-54.

[7] G. Serini, D. Ambrosi, E. Giraudo, A. Gamba, L. Preziosi, F. Bussolino. Modeling the early stages of vascular network assembly. EMBO J, 22 (2003) No. 8, 1771-1779.

[8] D. Berk, F. Yuan, M. Leunig, R. Jain. Fluorescence photobleaching with spatial fourier analysis: measurement of diffusion in light-scattering media. Biophys J, 65 (1993) No. 6 , 2428-2436.

[9] M. Chambard, J. Gabrion, J. Mauchamp. Influence of collagen gel on the orientation of epithelial cell polarity: follicle formation from isolated thyroid cells and from preformed monolayers. J Cell Biol, 91 (1981) No. 1, 157-166.

[10] T. Miura. Modulation of activator diffusion by extracellular matrix in turing system. RIMS Kokyuroku Bessatsu, B3 (2007), 165-176.

[11] F. Crick. Diffusion in embryogenesis. Nature, 225 (1970) No. 5231, 420-422.

[12] G. Reeves, C. Muratov, T. Schuepbach, S. Shvartsman. Quantitative models of developmental pattern formation. Dev Cell, 11 (2006) No. 3, 289-300.

[13] H. Buelow, O. Hobert. The molecular diversity of glycosaminoglycans shapes animal development. Annu Rev Cell Dev Biol, 22 (2006), 375-407.

[14] A. Okubo. Diffusion and ecological problems: mathematical models. Springer-Verlag, 1980. 
[15] M. Iida, M. Mimura, H. Ninomiya. Diffusion, cross-diffusion and competitive interaction. J Math Biol, 53 (2006), 617-641.

[16] C. Nicholson, E. Sykova. Extracellular space structure revealed by diffusion analysis. Trends Neurosci, 21 (1998) No. 5, 207-215.

[17] R. Thorne, C. Nicholson. In vivo diffusion analysis with quantum dots and dextrans predicts the width of brain extracellular space. Proc Natl Acad Sci U S A, 103 (2006) No. 14, 55675572.

[18] F. Gelain, D. Bottai, A. Vescovi, S. Zhang. Designer self-assembling peptide nanofiber scaffolds for adult mouse neural stem cell 3-dimensional cultures. PLoS ONE, 1 (2006):e119.

[19] P. Iglesias and P. Devreotes. Navigating through models of chemotaxis. Curr Opin Cell Biol, 20 (2008) No. 1, 35-40.

[20] I. Barkefors, S. Le Jan, L. Jakobsson, E. Hejll, G. Carlson, H. Johansson, J. Jarvius, J. Park, N. Jeon, J. Kreuger. Endothelial cell migration in stable gradients of vascular endothelial growth factor $a$ and fibroblast growth factor 2: effects on chemotaxis and chemokinesis. $\mathrm{J}$ Biol Chem, 283 (2008) No. 20, 13905-13912.

[21] J. Park, G. Keller, N. Ferrara. The vascular endothelial growth factor (vegf) isoforms: differential deposition into the subepithelial extracellular matrix and bioactivity of extracellular matrix-bound vegf. Mol Biol Cell, 4 (1993) No. 12, 1317-1326.

[22] C. Ruhrberg, H. Gerhardt, M. Golding, R. Watson, S. Ioannidou, H. Fujisawa, C. Betsholtz, D. Shima. Spatially restricted patterning cues provided by heparin-binding vegf-a control blood vessel branching morphogenesis. Genes Dev, 16 (2002) No. 20, 2684-2698. 